International Journal of Bifurcation and Chaos, Vol. 17, No. 10 (2007) 3691-3696

(C) World Scientific Publishing Company

\title{
A WAVELET TRANSFER MODEL FOR TIME SERIES FORECASTING
}

\author{
DAMIEN FAY \\ Department of Mathematics, National University of Ireland, \\ Galway, Ireland \\ damien.fay@nuigalway.ie \\ JOHN RINGWOOD \\ Department of Electronic Engineering, National University of Ireland, \\ Maynooth, Ireland \\ john.ringwood@eeng.nuim.ie
}

Received September 20, 2005; Revised April 3, 2006

\begin{abstract}
This paper is concerned with the case of an exogenous system in which a model is required to forecast a periodic output time series using a causal input. A novel approach is developed in which the wavelet packet transform is taken of both the dependent time series and causal input. This results in two sets of basis dictionaries and requires two bases to be chosen. It is proposed that the best bases to choose are those which maximize the mutual information. Input selection is then implemented by eliminating those coefficients of the selected input basis with low mutual information. As an example, a model is constructed to forecast short-term electrical demand.
\end{abstract}

Keywords: Wavelet packets; time-series; load forecasting.

\section{Introduction}

Time series forecasting is concerned with forecasting a dependant time series, $y(k)$, with a set of causal variables, $U(k)$, by using a model, $f(\cdot)$, as:

$$
y(k)=f(U(k))+\varepsilon(k)
$$

where $\varepsilon(k)$ is a residual term. However, estimation of $f(\cdot)$ is often a difficult task. This task may be aided by transforming the inputs and/or outputs into new domains prior to modeling as:

$$
A(y(k))=f^{\prime}(B(U(k)))+\varepsilon^{\prime}(k)
$$

where $A(\cdot)$ represents the output transform (or output filtering), $B(\cdot)$ represents the input transform (or input preprocessing), $\varepsilon^{\prime}(k)$ is a residual term (note: $\varepsilon^{\prime}(k) \neq \varepsilon(k)$ in general) and $f^{\prime}(\cdot)$ denotes the new model. The purpose of $B(\cdot)$ is to eliminate noncausal inputs and reduce multicollinearity (cross-correlation) in the inputs [Ljung, 1999]. The purpose of $A(\cdot)$ is to transform the dependent time series, $y(k)$, into a time series that is more correlated to the input. In addition, the distribution of the residual term is altered which can be advantageous, especially if the distribution of the original residual term, $\varepsilon(k)$, is non-Gaussian [Ljung, 1999].

Several types of transform have been applied in time series forecasting such as Principle Component Analysis (PCA) [Hiden et al., 1999], Independent Component Analysis (ICA) [Roberts et al., 2004], the Fourier Transform (FT) [Schoukens \& Pintelon, 1991], the Wavelet Transform (WT) [Yao et al., 2000] and the Wavelet Packet Transform (WPT) [Saito \& Coifman, 1997; Roberts et al., 2004; Milidiú et al., 1999; Nason \& Sapatinas, 2001] among others. However, the WT and WPT would seem ideal for time series forecasting as unlike PCA, ICA and the FT, some time information is preserved in the transformed variables. In addition, 


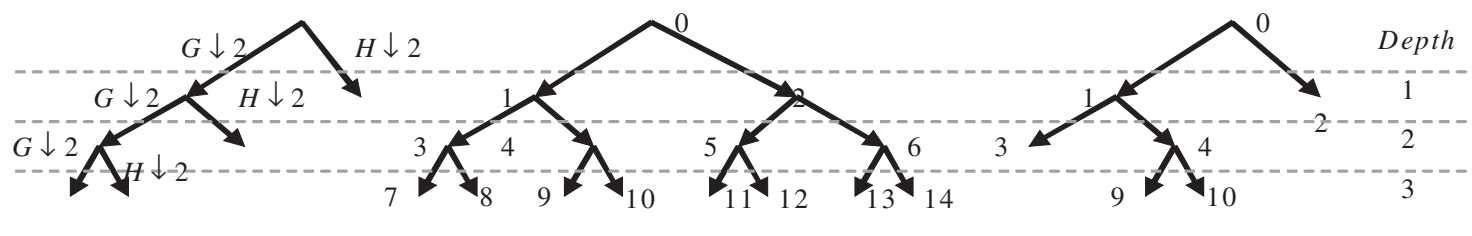

(a)

(b)

(c)

Fig. 1. Diagram of the WPT to a depth of three. (a) Packet $\{7,8,4,2\}$ (the wavelet transform), (b) the complete wavelet packet tree and labeled nodes, (c) example of another wavelet packet $\{3,9,10,2\}$.

the WPT allows an adjustable trade-off between time and frequency resolution in the transformed signal. The FT and WT have been used to transform both the input and output of a system prior to modeling [Schoukens \& Pintelon, 1991; Liu, 2005; Labat et al., 2000]. However, the WPT has not been widely used for this purpose. The wavelet transfer model proposed in this paper is similar to that proposed by Ramsey and Lampart [1998]. However, as the focus of this paper is on time series forecasting, several unique problems arise such as the joint selection of $A(\cdot)$ and $B(\cdot)$ (Sec. 3.1) and input reduction (Sec. 3.2).

\section{The Wavelet Packet Transform}

The WPT is implemented by successively filtering an input, $y(k)$ with specifically designed high pass, $H$, and low pass, $G$, filters forming a WPT tree (Fig. 1). This is followed by a down-sampling by two. ${ }^{1}$ As $H$ and $G$ form perfect reconstruction filters, the original data can be reconstructed from the down-sampled coefficients. With successive filtering, the level of frequency resolution increases at the expense of time resolution. As the option exists to filter each branch independently an adjustable timefrequency resolution trade-off is possible (three alternative trees or packets are shown in Fig. 1) (for an excellent textbook on wavelets see [Percival \& Walden, 1999]).

\section{The Wavelet Transfer Model}

The wavelet transfer model first prefilters the input and output using the wavelet packet transform. Input selection is then applied and a nonlinear model is used to relate the transformed input to the output as:

$$
A Y(k)=f\left(S^{\circ} B^{\circ} U(k)\right)+\varepsilon^{\prime}(k)
$$

where $A$ is a (WPT) basis transform of the output, $Y(k)=[y(k) y(k-1) \cdots y(k-s)], B$ is a (WPT) basis transform of the input, $U(k), S$ represents the shrinkage operator which reduces the dimensionality of the input (see Sec. 3.2), $f$ is a nonlinear function, $\varepsilon^{\prime}(k)$ is a vector of (filtered) error terms, ${ }^{2} s$ is the period of the data and ${ }^{\circ}$ denotes after.

\subsection{Packet selection technique}

Define:

$$
D_{1}=\left\{A_{i}\right\}_{i=1}^{N_{1}} \quad \text { and } \quad D_{2}=\left\{B_{j}\right\}_{j=1}^{N_{2}}
$$

where $D_{1}$ and $D_{2}$ are wavelet packet dictionaries of all possible WPT transforms of $Y(k)$ and $U(k)$, respectively. $A_{i}$ and $B_{j}$ are the elements of those dictionaries and $N_{1}$ and $N_{2}$ their respective lengths. The aim of packet selection is to choose an element of $D_{1}$ and $D_{2}$ jointly. It is proposed here to use the Mutual Information (MI, defined below) between the transformed input and output to determine the optimal transform:

$$
(A, B)=\underset{i, j}{\arg \max } I\left(A_{i} Y(k) ; B_{j} U(k)\right)
$$

where $A$ and $B$ are the bases to be chosen and $I(U ; Y)$ is the MI defined as:

$$
I(U ; Y)=\int_{Y} \int_{U} f_{U, Y}(u, y) \log \frac{f_{U, Y}(u, y)}{f_{U}(u) f_{Y}(y)} d u d y
$$

where $f_{U}(u)$ and $f_{Y}(y)$ are the (multivariate) probability distributions of $U$ and $Y$ respectively.

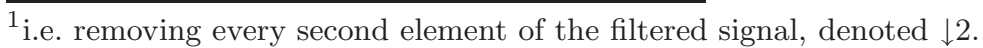

${ }^{2}$ Note that $f(\cdot)$ makes a forecast of the transformed output, $\hat{Y}^{\prime}(k)$, and not of $Y(k)$. Typically $f(\cdot)$ will be trained to minimize some cost function (e.g. the Mean Squared Error, MSE) of the forecast errors. However, in this case $f(\cdot)$ minimizes the cost function with respect to $\varepsilon^{\prime}(k)$ and not $\varepsilon(k)$. This is sometimes advantageous [Ljung, 1991] as it may remove disturbances at high and low frequencies that are not wanted during modeling.
} 
$f_{U, Y}(u, y)$ is the joint PDF between $U$ and $Y$. Saito et al. [2002] proposed a Local Discriminant Basis (LDB) algorithm for calculating the MI for a classification problem. However, estimating $f_{U, Y}(u, y)$ for multivariate continuous data is a difficult task [Darbellay, 1999]. An approximation of the MI may be made by means of multivariate Gaussian kernels as [Nilsson et al., 2002]:

$$
\begin{aligned}
I(U ; Y) \approx & \sum_{j=1}^{M} \int_{Y} \int_{U} \alpha_{j} G_{j_{U, Y}}(u, y) \\
& \times \log \frac{G_{j_{U, Y}}(u, y)}{G_{j_{U}(u)} G_{j_{Y}(y)}} d u d y \\
& \sum_{j=1}^{M} \alpha_{j}=1
\end{aligned}
$$

where $G_{j_{U, Y}}(u, y), G_{j_{U}(u)}, G_{j_{Y}(y)}$ are multivariate Gaussian distributions for the $j$ th kernel, $M$ denotes the number of modes in the approximated distributions and $\alpha_{j}$ is the $j$ th weight associated with each kernel to ensure that the total probability equals one. The optimum mean and covariance matrices for the kernels may be estimated using the Expectation Maximization (EM) algorithm [Dempster et al., 1977]. Given a Gaussian kernel the expression for the approximate MI then reduces to:

$$
I(U ; Y) \approx \frac{1}{2} \sum_{j=1}^{M} \alpha_{j} \log \frac{\left|\hat{C}_{j_{U Y}}\right|}{\left|\hat{C}_{j_{U}}\right|\left|\hat{C}_{j_{Y}}\right|}
$$

where $|\cdot|$ denotes the determinant, $\hat{C}_{j_{U Y}}, \hat{C}_{j_{Y}}$ and $\hat{C}_{j_{U}}$ are the sample cross and auto-covariance matrices of the $j$ th kernel.
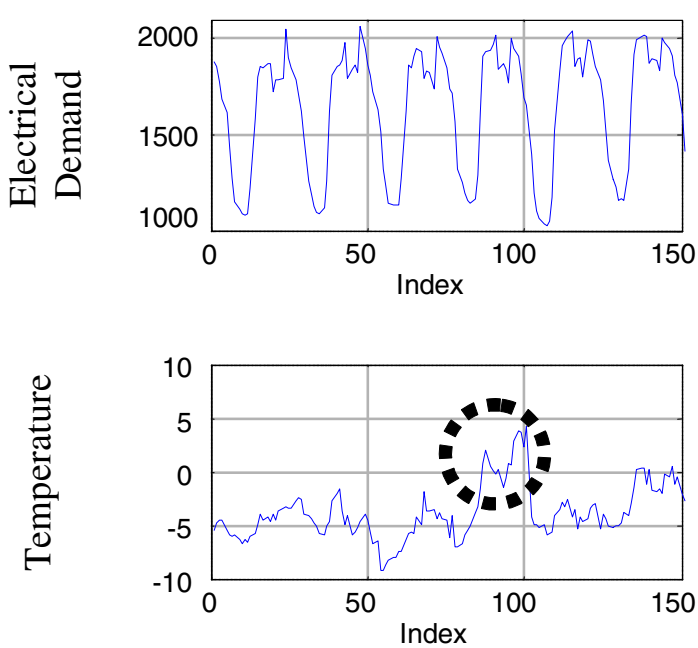

\subsection{Input selection}

Input selection requires reduction in the dimension of $B U(k)$. Typically, a threshold is used in which wavelet coefficients with mutual information (or entropy in the univariate case) below the threshold are eliminated [Percival \& Walden, 2000]. However, the purpose here is to reduce the dimension of the input space to a specific size. Given $A$ and $B$ (calculated in Sec. 3.1), input selection is implemented by retaining those variables that individually have the highest mutual information with the output as:

$$
\begin{aligned}
U^{\prime \prime}= & \left\{A u_{j_{m}}: m=1, \ldots, N_{\operatorname{dim}} / \underset{j_{m} \in\left\{i / j_{m-1}, \ldots, j_{1}\right\}}{\arg \max }\right. \\
& \times \hat{I}\left(A u_{j_{m}} ; B Y\right)
\end{aligned}
$$

where $U^{\prime \prime}$ is the reduced input set of dimension $N_{\text {dim }}, A u_{l}$ is the $l$ th element of $A U$ and $j_{m}$ are the indices of the retained elements. $\hat{I}\left(A u_{l} ; B Y\right)$ is estimated as in Eq. (8).

\section{Example Application: Hourly Electricity Demand Forecasting}

Hourly electrical demand is a time series driven by human activity which is influenced by weather; temperature and humidity being the dominant causal variables. The data spans the years 1986-2000, only Mondays to Fridays and only the months January to March. In addition, this data has been detrended. The data has been split into three different groups for analysis; training set $(400 \times 24$ points $)$, validation set $(170 \times 24$ points $)$ and test set $(170 \times 24$ points). Finally, note that this data is periodic with a period of 24 (hours) and that full details
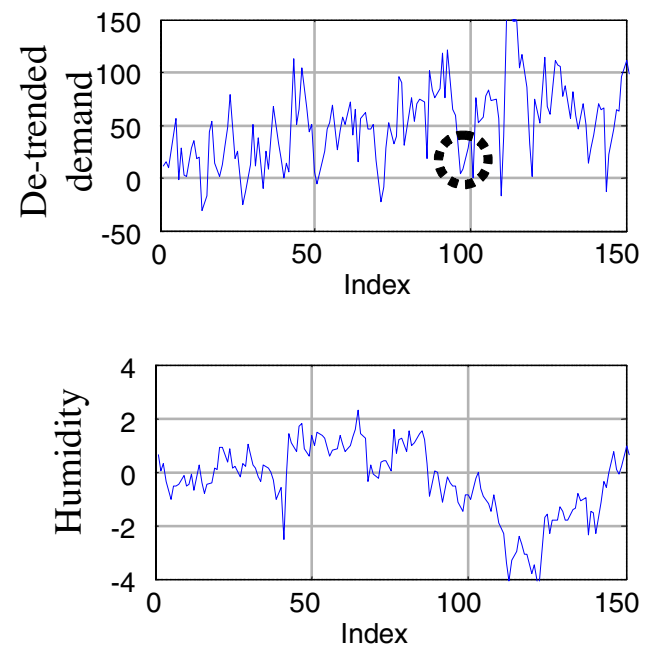

Fig. 2. Graph of original and detrended electrical demand, temperature and humidity. 


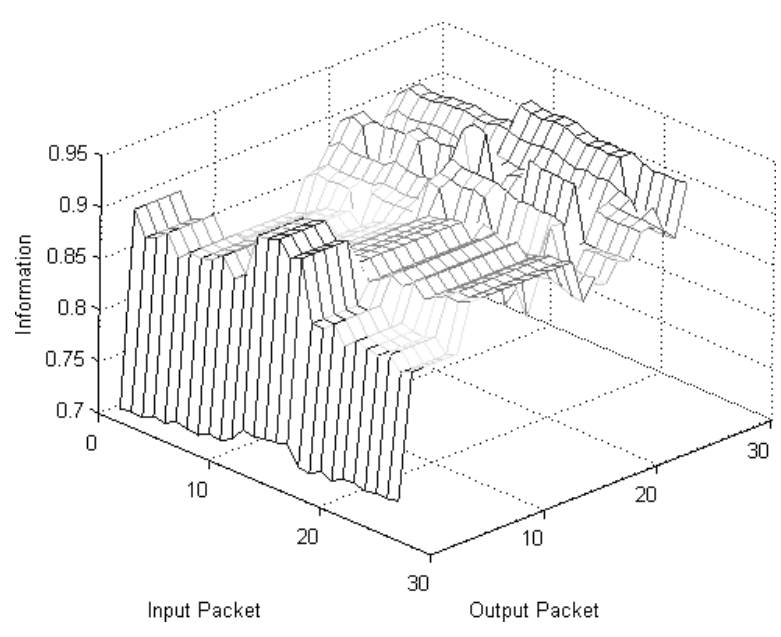

(a)

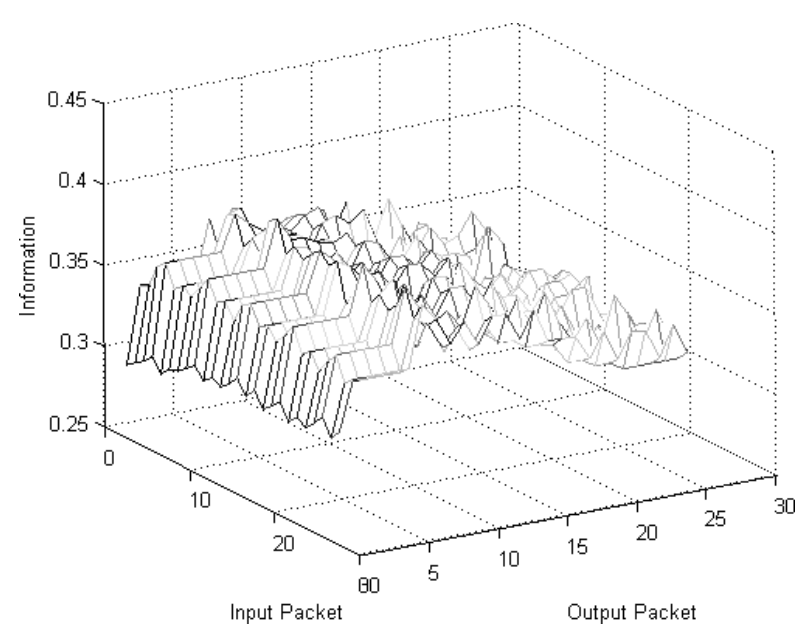

(b)

Fig. 3. Graph of mutual information between detrended input and (a) temperature, (b) humidity.

of the above can be found in [Fay et al., 2003]. In Fig. 2 a rise in temperature from indices 87:100 and a corresponding fall in the detrended demand at indices 95:100, are indicated. This example suggests that a low frequency component in the temperature (i.e. the average temperature between indices 87:100) causes a corresponding change in the dependant variable but at a later time and for a shorter period. Thus, the wavelet transfer model would seem ideal in identifying these time-frequency correlations between the input and output.

For the purposes of this paper the output time series is the detrended demand, $y(k)$, and there are two inputs, temperature and humidity, denoted $u^{t}(k)$ and $u^{h}(k)$ respectively. The WPT to a depth of four is taken of $y(k), u^{t}(k)$ and $u^{h}(k)$ using Daubechie's "D4" wavelet [Percival \& Walden, 2000], giving three dictionaries $D_{1}, D_{2}^{1}$ and $D_{2}^{2}$. $Y(k), U^{t}(k)$ and $U^{h}(k)$ are constructed as:

$$
\begin{aligned}
Y(k) & =[y(k) \cdots y(k-24)] \\
U^{t}(k) & =\left[u^{t}(k) \cdots u^{t}(k-72)\right] \\
U^{h}(k) & =\left[u^{h}(k) \cdots u^{h}(k-72)\right] \quad k=24,48, \ldots
\end{aligned}
$$

Note that $U^{t}(k)$ and $U^{h}(k)$ contain weather data up to a lag of three days (72 hours). After three days, it is considered that the weather has no effect on the demand [Fay et al., 2003]. In addition, note that as the data is periodic, it is sufficient to take every 24 th value of $k .^{3}$ The input selection reduces the number of input variables to seven. ${ }^{4}$ Figure 3 shows the mutual information between the inputs and outputs for different packet transforms, calculated using $M=1$ (this is equivalent to using the correlation).

Table 1, below, summarizes the optimal packets chosen for the input-output in Fig. 3. As can be seen, the transformed temperature has higher mutual information with the transformed detrended load, and so it is chosen as the transform to be applied.

The next stage is to model $A Y(k)$ with $B U(k)$ using a feed-forward neural network. The network used is similar to that described in [Fay et al., 2003] (the inputs differ) and so it is not described here. For comparison, the Wavelet Transfer Model (WTM) is compared to a Transfer Model (TM) in

Table 1. Packet transforms that share the maximum mutual information with detrended load.

\begin{tabular}{lccccc}
\hline Variable & $\begin{array}{c}\text { Input Packet } \\
\text { Number }\end{array}$ & $\begin{array}{c}\text { Input Packet } \\
\text { Nodes }\end{array}$ & $\begin{array}{c}\text { Output Packet } \\
\text { Number }\end{array}$ & $\begin{array}{c}\text { Output Packet } \\
\text { Nodes }\end{array}$ & $\begin{array}{c}\text { Mutual } \\
\text { Information }\end{array}$ \\
\hline Temperature & 26 & $\{3,9,10,2\}$ & 12 & $\{7,8,9,10,11,12,6\}$ & 0.9455 \\
Humidity & 7 & $\{3,4,5,13,14\}$ & 9 & $\{3,4,2\}$ & 0.3901 \\
\hline
\end{tabular}

\footnotetext{
${ }^{3}$ i.e. the data is arranged by day, see Eq. (10).

${ }^{4}$ This number is chosen subjectively with experience.
} 
Table 2. A comparison of the wavelet transfer model and a model without the WPT.

\begin{tabular}{cccc}
\hline Model & PMSE Training Set & PMSE Validation Set & PMSE Novelty Set \\
\hline WTM & 3586 & 5236 & 6815 \\
TM & 3929 & 5876 & 7569 \\
\hline
\end{tabular}

which the WPT is not applied, i.e. $A=1, B=1$ (note: input selection is still applied). Table 2 summarizes the results.

\section{Conclusions}

A novel method was presented for modeling nonlinear exogenous time series based on the wavelet packet transform. For a nonlinear system, an input at one frequency will result in an output at different frequencies. However, estimating frequency information requires the use of a window. As the window size increases the frequency estimate is improved but time resolution is decreased, as the window now covers a larger segment of time. As mentioned in the introduction, the wavelet packet transform allows an adjustable trade-off of time-frequency resolution. The aim of the WTM is to find those (wavelet packet) transformed domains that give the clearest relationship (in the mutual information sense) between the input and output. Training a nonlinear model can be a difficult task. However, it is proposed that the training process is easier in the WTM transformed domains.

However, like all black-box modeling techniques, the suitability of this technique is data dependent. There is no means a priori of determining if this technique is applicable other than with experience. For the electricity demand data used in the example, it was known from experience that a low frequency component in temperature affects a low frequency component in demand. When compared to a model trained using untransformed data the WTM approach performed better (Table 2). As can be seen in Table 2, the Prediction Mean Squared Error (PMSE) is consistently lower for the WTM across all three data sets. Based on these empirical results the WTM would appear to be superior for the task of electricity demand forecasting.

In implementing this approach there are several arbitrary elements. The choice of the wavelet basis function depends again on experience. A Haar wavelet basis function is equivalent to differencing [Percival \& Walden, 1999] and in this case the WTM is equivalent to the well-known Box-Jenkins transfer model [Ljung, 1999]. For the example application, a Daubechie's " $D 4$ " wavelet was chosen as this has a support of four (hours) which is thought (from experience) to be appropriate for hourly electricity demand forecasting. The technique for estimating the mutual information depends on what is known about the underlying distribution of the data. Given no prior knowledge, a nonparametric technique is proposed (Sec. 3.1).

Perhaps the most serious drawback of the WTM technique is the way the dictionary size increases with depth. The number of elements for a dictionary of depth $N$ may be calculated recursively as:

$$
s(n)=(s(n-1)+1)^{2} \quad n=1,2, \ldots, N
$$

where $s(N)$ is the number of elements of a dictionary of depth, $N$ and $s(1)=1$. As $s(N)$ increases rapidly with $N$, it is impossible to evaluate all packets in a dictionary individually for all but the smallest depths [Coifman \& Wickerhauser, 1992]. Coifman and Wickerhauser [1992] instead proposed using an additive measure such as entropy which allows the evaluation of each binary branch individually thus reducing the number of operations from $\mathrm{O}\left(N(\log N)^{2}\right)$ to $\mathrm{O}(N)$. However, mutual information is not an additive measure and so the WTM technique is restricted to low values of $N(N=4$ would appear to be the practical limit).

\section{Acknowledgment}

The authors would like to thank the Irish national grid operator (EirGrid Plc).

\section{References}

Coifman, R. R. \& Wickerhauser, M. V. [1992] "Entropy based algorithms for best basis selection," IEEE Trans. Inform. Th. 38, 713-718.

Darbellay, G. A. [1999] "An estimator of the mutual information based on a criterion for independence," Comput. Stat. Data Anal. 32, 1-17.

Dempster, A. P., Laird, N. M. \& Rubin, D. B. [1977] "Maximum likelihood from incomplete data via the EM algorithm," J. Roy. Stat. Soc., Series B 39, 1-38. 
Fay, D., Ringwood, J. V., Condon, M. \& Kelly, M. [2003] "24-hour electrical load data - A sequential or partitioned time series?" Neurocomputing 55, 469-498.

Hiden, H. G., Willis, M. J., Tham, M. T. \& Montague, G. A. [1999] "Non-linear principal components analysis using genetic programming," Comput. Chem. Engin. 23, 413-425.

Labat, D., Ababou, R. \& Mangin, A. [2000] "Rainfallrunoff relations for karstic springs. Part II: Continuous wavelet and discrete orthogonal multiresolution analyses," J. Hydrol. 238, 149-178.

Liu, L. T., Hsu, H. T. \& Grafarend, E. W. [2005] "Wavelet coherence analysis of length-of-day variations and El Niňo-southern oscillation," J. Geodyn. 39, 267-275.

Ljung, L. [1999] System Identification: Theory for the User, 2nd edition (Prentice Hall, NJ).

Mallat, S. G. [1989] "A theory for multiresolution signal decomposition: The wavelet representation," IEEE Trans. Patt. Anal. Mach. Intell. 11, 674-693.

Milidiú, R. L., Machado, R. J. \& Rentería, R. P. [1999] "Time series forecasting through wavelets transformation and a mixture of expert models," Neurocomputing 28, 145-156.

Nason, G. P. \& Sapatinas, T. [2001] "Wavelet packet transfer function modelling of nonstationary time series," Stat. Comput. 12, 45-56.

Nilsson, M., Gustafsson, H., Andersen, S. V. \& Kleijn, W. B. [2002] "Gaussian mixture model based mutual information estimation between frequency bands in speech," IEEE Int. Conf. Acoust. Speech Sign. Process. 1, pp. 525-528.

Percival, D. B. \& Walden, A. T. [2000] Wavelet Methods for Time Series Analysis (Cambridge University Press, Cambridge).

Ramsey, J. B. \& Lampart, C. [1998] "The decomposition of economic relationships by time scale using wavelets: Expenditure and income," Stud. Nonlin. Dyn. Economet. 3, 23-42.

Roberts, S., Roussos, E. \& Choudrey, R. [2004] "Hierarchy, priors and wavelets: Structure and signal modelling using ICA," Sign. Process. 84, 283-297.

Saito, N. \& Coifman, R. R. [1997] "Extraction of geological information from acoustic well-logging waveforms using time-frequency wavelets," Geophysics 62, 19211930.

Saito, N., Coifman, R. R., Geshwind, F. B. \& Warner, F. [2002] "Discriminant feature extraction using empirical probability density estimation and a local basis library," Patt. Recogn. 35, 2841-2852.

Schoukens, J. \& Pintelon, R. [1991] Identification of Linear Systems: A Practical Guideline to Accurate Modeling (Pergamon Press, London).

Yao, S. J., Song, Y. H., Zhang, L. Z. \& Cheng, X. Y. [2000] "Wavelet transform and neural networks for short-term electrical load," Energy Conver. Manag. 41, 1975-1988. 